\title{
(2) OPEN ACCESS \\ What do patients with glaucoma see: a novel iPad app to improve glaucoma patient awareness of visual field loss
}

\author{
Meghal Gagrani (D) , 1 Jideofor Ndulue, ${ }^{1}$ David Anderson, ${ }^{1}$ Sachin Kedar, ${ }^{2}$ Vikas Gulati, ${ }^{1}$ \\ John Shepherd, ${ }^{1}$ Robin High, ${ }^{3}$ Lynette Smith, ${ }^{3}$ Zachary Fowler, ${ }^{4}$ Deepak Khazanchi, ${ }^{4}$ \\ Mark Nawrot, ${ }^{5}$ Deepta Ghate ${ }^{1}$
}

- Supplemental material is published online only. To view please visit the journal online (http://dx.doi.org/10.1136/ bjophthalmol-2020-317034).

${ }^{1}$ Ophthalmology and Visual Sciences, University of Nebraska Medical Center, Omaha, Nebraska, USA

${ }^{2}$ Ophthalmology and Visual Sciences, Department of Neurological Sciences, University of Nebraska Medical Center, Omaha, Nebraska, USA ${ }^{3}$ College of Public Health, University of Nebraska Medical Center, Omaha, Nebraska, USA ${ }^{4}$ College of Information Science and Technology, University of Nebraska at Omaha, Omaha, Nebraska, USA

${ }^{5}$ Department of Psychology, North Dakota State University, Fargo, North Dakota, USA

Correspondence to Deepta Ghate, Stanley M. Truhlsen Eye Institute, University of Nebraska Medical Center, Omaha, NE 68105, USA;

dghate@unmc.edu

Received 7 June 2020 Revised 22 September 2020 Accepted 22 October 2020

Check for updates

(C) Author(s) (or their employer(s)) 2020. Re-use permitted under CC BY-NC. No commercial re-use. See rights and permissions. Published by BMJ.

To cite: Gagrani M, Ndulue $\mathrm{J}$, Anderson $\mathrm{D}$, et al. $\mathrm{Br}$ Ophthalmol Epub ahead of print: [please include Day Month Year]. doi:10.1136/ bjophthalmol-2020-

317034

\begin{abstract}
Purpose Glaucoma patients with peripheral vision loss have in the past subjectively described their field loss as 'blurred' or 'no vision compromise'. We developed an iPad app for patients to self-characterise perception within areas of glaucomatous visual field loss.
\end{abstract}

Methods Twelve glaucoma patients with visual acuity $\geq 20 / 40$ in each eye, stable and reliable Humphrey Visual Field (HVF) over 2 years were enrolled. An iPad app (held at $33 \mathrm{~cm}$ ) allowed subjects to modify 'blur' or 'dimness' to match their perception of a $2 \times 2 \mathrm{~m}$ wall-mounted poster at $1 \mathrm{~m}$ distance. Subjects fixated at the centre of the poster (spanning $45^{\circ}$ of field from centre). The output was degree of blur/dim: normal, mild and severe noted on the iPad image at the 54 retinal loci tested by the HVF 24-2 and was compared to threshold sensitivity values at these loci. Monocular (Right eye (OD), left eye (OS)) HVF responses were used to calculate an integrated binocular (OU) visual field index (VFI). All three data sets were analysed separately.

Results 36 HVF and iPad responses from 12 subjects (mean age $71 \pm 8.2 \mathrm{y}$ ) were analysed. The mean VFI was $77 \%$ OD, $76 \%$ OS, $83 \%$ OU. The most common iPad response reported was normal followed by blur. No subject reported dim response. The mean HVF sensitivity threshold was significantly associated with the iPad response at the corresponding retinal loci (For OD, OS and $\mathrm{OU}$, respectively $(\mathrm{dB})$ : normal: $23,25,27$; mild blur: 18 , 16, 22; severe blur: $9,9,11)$. On receiver operative characteristic (ROC) curve analysis, the HVF retinal sensitivity cut-off at which subjects reported blur was 23.4 OD, 23 OS and $23.3 \mathrm{OU}(\mathrm{dB})$.

Conclusions Glaucoma subjects self-pictorialised their field defects as blur; never dim or black. Our innovation allows translation of HVF data to quantitatively characterise visual perception in patients with glaucomatous field defects.

\section{INTRODUCTION}

Glaucoma is a progressive optic neuropathy and second leading cause of blindness worldwide ${ }^{1}$ with an estimated prevalence of $2 \%$ in the United States population over age 40 years. $^{2}$ Glaucoma is a 'silent disease' and 41-50\% of all newly diagnosed glaucoma patients in western countries already have moderate-advanced visual field loss at diagnosis. ${ }^{3} 4$ This loss affects activities of daily living in glaucoma and can increase the risk of motor vehicle crashes 56 and falls. ${ }^{7}$ Clinical assessment of glaucoma focuses on intraocular pressure management and structural and functional assessment of optic nerve damage; yet, patients' perception of their own vision loss is not generally assessed in a standard way. In this vein, 66\% American glaucoma specialists surveyed reported that 'patients do not convey problems or are in denial about vision loss' or there is 'not enough time to address low vision issues during clinic visit'.

Our understanding of visual perception in glaucoma is extrapolated from vision science studies in the normal population on physiologic and simulated blind spots, 910 and qualitative descriptive studies in the glaucoma population. ${ }^{11} 12$ Glaucoma subjects have described their visual symptoms as 'missing', 'blur' and 'needing more light' ${ }^{11}$ and their scotomas as blurred/missing/grey $(76 \%)$ or normal $(19 \%))^{13}$ In a forced choice experiment, subjects with moderate-advanced glaucoma were asked to select images that most represents their perception of field loss. Sixty-seven per cent chose the picture that displayed 'blurred' and 'missing' parts while $26 \%$ chose the 'normal picture'. ${ }^{12}$

The sparse literature on this subject has translated to inconsistent physician understanding and poor patient education literature on glaucomatous field defects. The American Academy of Ophthalmology patient education website ${ }^{14}$ depicts glaucomatous vision loss as 'large arcuate black holes' which is inconsistent with literature ${ }^{4} 12$ or patient complaints in glaucoma clinic.

Our study had two objectives: the primary objective was to develop an iPad-based application for glaucoma subjects to pictorialise their vision field loss that can answer the question 'what do patients see in the areas of their scotomas'. The secondary objective was to validate the application by correlating subjective responses with parameters obtained on automated perimetry (Humphrey visual fields (HVF)). We hypothesised that subjects with glaucoma could observe and map the discrepancy between their visual perception on a large $2 \mathrm{~m} \times 2 \mathrm{~m}$ wall-mounted scene (projecting to $45^{\circ}$ of field around fixation at $1 \mathrm{~m}$ ) and a handheld copy of the scene on the iPad at $33 \mathrm{~cm}$ (projected to central preserved vision).

A quantitative study of the association between visual field thresholds and perceptual deficits would advance our understanding of the psychophysiology 
of vision loss. An enhanced understanding of glaucomatous visual disability by physicians, patients and caregivers will translate to increased utilisation of low vision rehabilitation strategies by glaucoma patients, increased empathy by caregivers and physicians and improved patient-centred care.

\section{METHODS}

\section{Study design}

This was a cross-sectional study designed to investigate the subjective perception of visual field loss in patients with glaucoma. The study was approved by the university Institutional Review Board and followed the tenets of the Declaration of Helsinki.

\section{Subjects}

Subjects were recruited from the glaucoma clinic at the Truhlsen Eye Institute at the University of Nebraska Medical Centre. A written informed consent was obtained from all subjects.

We included subjects diagnosed with glaucoma. The diagnosis was made by a glaucoma specialist based on characteristic optic nerve head appearance and visual field defects. Inclusion criteria were best corrected visual acuity of 20/40 or better in both eyes and a stable visual field defect over the last 2 years (24-2 or 30-2), Swedish Interactive Threshold Algorithm (SITA) HVF (Carl Zeiss Meditec Inc., Dublin, CA) and a Montreal Cognitive Assessment (MoCA) score of 25 and above. Visual field stability was defined by the treating glaucoma provider based on global indices and pattern deviation plot.

We excluded subjects with (a) poor reliability indices on HVF (>20\% false positives, false negatives or fixation losses) and (b) non-glaucomatous causes of field loss (retinal disease, central nervous system disease or other optic neuropathies)

Poster design: A $2 \mathrm{~m}$ x $2 \mathrm{~m}$ poster depicting a busy naturalistic street scene was designed in collaboration with a graphic design expert (figure 1 ) to subtend a visual angle of $45^{\circ}$ at $1 \mathrm{~m}$ around the yellow fixation target in the centre. The naturalistic scene was populated by small objects placed in the areas of common glaucomatous visual field defects (figure 1A,B). Standard room lighting was utilised during the experiment. We used a standard ophthalmology examination room to conduct the experiment and a luminance metre (Konica Minolta Sensing AmericasInc., USA LS150) was used to measure the illumination. The light source of the exam room emitted 3500 lux and the wall reflected 52 lux in illumination. The maximum and minimum reflectance off the poster was 2.6 and 50.3 lux, respectively. As a comparison, the HVF bowl has a background luminance of 31.5 apostilb, which is equivalent 10 lux. ${ }^{15}$

App development (figure 1B): the app was developed and used in the 11" iPad-4 model using the latest iOS software available (iOS 12.3). The app interface allowed subjects to select parts of the scene on the device and modify blur and dimness (or contrast) as visualised on the poster. The value of each effect was scaled for blur and dimness into 0-10 integer values.

The app gives an output in a grid format (figure 1C) where each point on the grid corresponded to a specific locus on HVF 24-2 loci (for example, $3^{\circ}, 9^{\circ}, 15^{\circ}, 21^{\circ}$ and $27^{\circ}$ from vertical and horizontal meridians). The app gives us a $0-10$ value for blur and dimness for each retinal loci depending on patient response. These values were stored in separate data files for each measure.

App administration (figure 1D,E): an investigator masked to the subjects' visual field defects administered the study monocularly to the right eye, left eye and then binocularly in the same sequence. When testing monocularly, the other eye was covered with an eye patch. The subjects used their own bifocal refractive

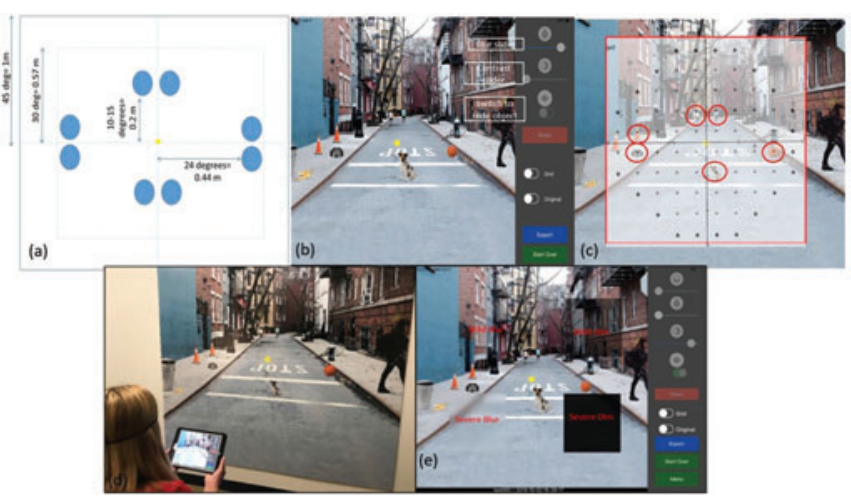

Figure 1 Building and administering the app. (A) Poster design: a $2 \times 2 \mathrm{~m}$ poster subtending a visual angle of $45^{\circ}$ at $1 \mathrm{~m}$ was designed depicting a naturalistic street scene with a central fixation point (yellow dot) and objects of interest placed at areas of common glaucomatous visual field defect (blue dots in 1a and red circles in $1 \mathrm{c}$ ). The objects of interest subtended a visual angle of $5-7^{\circ}$ at $1 \mathrm{~m}(10-12 \mathrm{~cm}$ on the poster) with good contrast from the surroundings. (B) Screenshot of iPad app: areas can be selected and modified using a sliding scale for blur and contrast. A toggle switch can be used to hide an object. (C) Analysis: the subject response at each point on the poster corresponding to the HVF loci was recorded as visualised in $1 \mathrm{c}$. There were 54 loci tested monocularly for each HVF 24-2 and 56 loci for HVF 30-2 and for the binocular fields. (D) Subject compares poster with iPad image and modifies the iPad image until it matches their perception of the poster. (E) Screenshot of iPad app showing the varying degrees of blur and dimness adjusted by the sliders present on the right ( ${ }^{*}$ The topmost slider is an extra Colour slider included in the latest version of the app which was not used for this study). HVF, Humphrey visual fields.

correction for the experiment. For viewing the poster, the subjects used their distance correction; for viewing the iPad image, the subjects used their near correction. The subjects were seated $1 \mathrm{~m}$ from the poster and held the iPad at a distance of $33 \mathrm{~cm}$. The subjects were instructed to look at the fixation target on the poster throughout the test and compare each quadrant of the poster to the image on the iPad (figure 1D). The subjects were asked to fixate on the central fixation point of the poster but were instructed to scan the iPad image freely. They were asked to modify the contrast and blur on the iPad using a sliding scale for each sector (figure 1E), until the discrepancy between the poster and the iPad image was eliminated. The subjects could modify the image on the iPad until the two images appeared to match and they replied in the affirmative to the query 'Does the iPad image match what you see when you look up at the poster?'. If the subjects replied in the negative, the iPad image was further modified until they confirmed that the two images matched (figure 2). It took approximately 8-10 min to complete the task with each eye and none of the subjects abandoned the test because of exhaustion.

\section{Data analysis}

Creating an integrated visual field and calculation of binocular visual field index (VFI): an integrated binocular visual field was created using the subject's most recent right and left HVF, using the standard binocular summation method described by NelsonQuigg et al. ${ }^{16}$ Binocular VFI was then calculated using the method described by Bengtsson and Heijl. ${ }^{17}$ The online supple mental file (online appendix) has further details on the calculations used. 

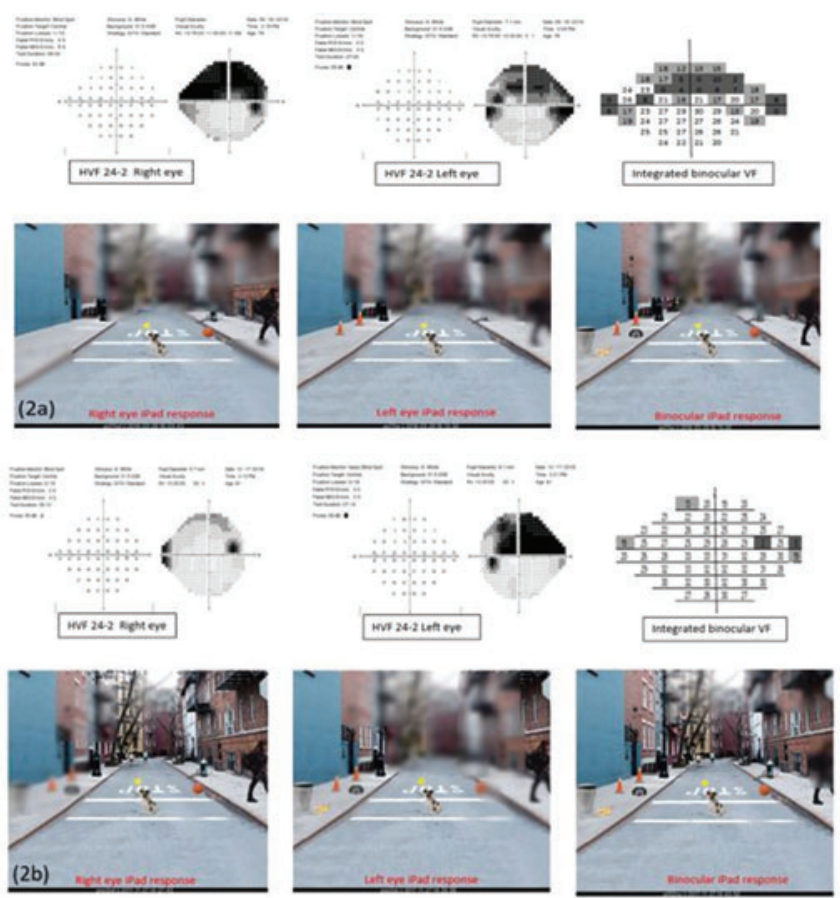

Figure 2 (A, B) Example of monocular and binocular Humphrey visual fields (HVF 24-2) for two subjects with the corresponding subject response on the iPad app. For for example, (A) Subject 1: left eye: the basketball corresponds to $24-27^{\circ}$ of visual angle horizontally to the right and $4-7^{\circ}$ inferiorly which is seen missing from the left eye response corresponding to the inferior nasal step in the left eye.

Extraction of data from iPad app: data from the iPad application were recorded on Microsoft Excel (Microsoft Corporation) spreadsheet programme. Right eye, left eye and binocular responses were recorded individually. The dimness and blur responses on the poster were recorded at the points corresponding to the HVF retinal loci (figure 1C). These values were graded on two parallel scales as normal (a value of 0 ), mild blur or dimness (1-5) or severe blur (6-10 or missing object) or severely $\operatorname{dim}(6-10)$. The data were not analysed as a continuous variable due to a high degree of heteroscedasticity. The coded iPad poster values were compared to threshold sensitivity values on the HVF at corresponding locations.

Statistical analysis: all statistical analyses were performed using SAS/STAT software, Version 9.4 (SAS Institute Inc., Cary, NC, USA). A linear mixed model with a random subject effect was applied to compute the means and 95\% CIs of the retinal sensitivity threshold values for normal, mild and severe blurred images. The means for HVF (right-eye (OD), left-eye (OS) and binocular (OU)) were compared to the means from the corresponding retinal loci from the pictorial responses from the 12 subjects by computing the differences in these means within each group. The analyses were conducted separately for right eye, left eye and both eyes.

HVF retinal sensitivity threshold values were stratified into four groups: $0-10 \mathrm{~dB}, 11-20 \mathrm{~dB}, 21-30 \mathrm{~dB}$ and $>30 \mathrm{~dB}$. The association between these four categories of HVF retinal sensitivity and the categories of pictorial response (normal, mild and severe blur) was tested with logistic regression, the approach applied with a small number of discrete outcomes.

The pictorial response from the poster was then recoded as 'normal' versus 'not normal'. Graphs of the receiver operative characteristic (ROC) curves were produced along with areas under these curves (AUC) to determine the HVF cut-off value to maximise the sensitivity and specificity for an 'abnormal' iPad response.

\section{RESULTS}

Twelve subjects with 36 visual fields (12 OD, 12 OS and 12 integrated binocular) were included in the study. There were eight females and four males with a mean age of $71 \pm 8.2$ years. The MoCA score ranged from 25 to 30 with a mean of $26.9 \pm 1.5$. The monocular VFI on the HVF (11 with 24-2 HVF fields and 1 with 30-2 HVF fields) ranged from 26 to 100 . The HVF-VFI (mean \pm SD) was $76.7 \pm 21.8 \mathrm{OD}$ and $75.9 \pm 20.9$ OS and 83.1 \pm 15.5 binocularly.

iPad response: all the glaucoma subjects reported some degree of blur. None of the subjects used the dim or blackening response on the iPad. The iPad responses at the retinal loci tested were categorised as normal (74\% OD, 81\% OS, 90\% OU), mild blur (14\% OD, $8 \%$ OS and 4\% OU) and severe blur (12\% OD, 11\% OS and $6 \%$ OU).

\section{iPad response and HVF threshold sensitivity}

The HVF sensitivity threshold (figure 3 ) was significantly associated with the iPad response in the three data sets studied (all $\mathrm{p}<0.001)$. The mean HVF threshold was $23 \mathrm{~dB}$ OD, $25 \mathrm{~dB}$ OS, $27 \mathrm{~dB}$ OU for a normal response, $18 \mathrm{~dB}$ OD, $16 \mathrm{~dB}$ OS, $22 \mathrm{~dB}$ OU for mild blur and $9 \mathrm{~dB}$ OD, $9 \mathrm{~dB}$ OS, $11 \mathrm{~dB}$ OU for severe blur.

Table 1 tabulates the iPad response on the poster given a particular $\mathrm{dB}$ value on the HVF.

For retinal loci with $\leq 10 \mathrm{~dB}$ on HVF, most subjects reported mild or severe blur (64\% OD, 61\% OS, 50\% OU). For retinal loci with $>20 \mathrm{~dB}$ on HVF, most subjects reported normal vision $(87 \%$ OD, $91 \%$ OS, $96 \%$ OU). For retinal loci with $>30 \mathrm{~dB}$ on HVF, almost all the subjects reported normal vision on the iPad $(96 \%$ OD, 100\% OS, 98\% OU). These observed differences between different categories of pictorial response were statistically significant for OD, OS and OU $(\mathrm{p}<0.001)$.

ROC curve analysis determined that the optimal cut-off HVF retinal sensitivity threshold value at which subjects reported 'blur' in their visual field was $23.4 \mathrm{~dB}(\mathrm{OD}), 23 \mathrm{~dB}(\mathrm{OS})$ and $23.3 \mathrm{~dB}(\mathrm{OU})$. The area under the ROC curve was $0.80 \mathrm{OD}, 0.81$

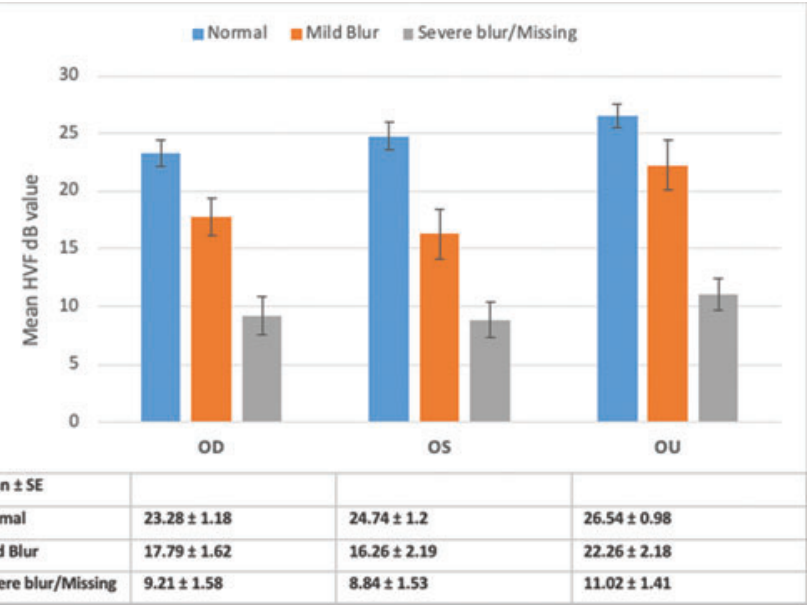

Figure 3 Mean HVF retinal sensitivity threshold value $(\mathrm{dB})$ was significantly associated with subject response on the iPad app. $d B$, decibels; HVF, Humphrey visual fields; OD, right eye; OS, left eye, OU, both eyes; SE, standard error. 


\begin{tabular}{|c|c|c|c|c|}
\hline HVF & Normal & Mild blur & Severe blur/missing & Total \\
\hline \multicolumn{5}{|c|}{ OD (count, row \%) } \\
\hline $0-10 \mathrm{db}$ & $49(36.0)$ & $30(21.8)$ & $58(42.6)$ & $137(100)$ \\
\hline $11-20 \mathrm{db}$ & $49(69.1)$ & $17(23.3)$ & $7(19.1)$ & $73(100)$ \\
\hline $21-30 \mathrm{db}$ & 317 (85.9) & $43(11.6)$ & $10(2.7)$ & $370(100)$ \\
\hline$>30 \mathrm{db}$ & $67(95.7)$ & $1(1.4)$ & $2(2.8)$ & $70(100)$ \\
\hline Total & $482(74.3)$ & $91(14)$ & $77(11.8)$ & $650(100)$ \\
\hline \multicolumn{5}{|c|}{ OS (count, row \%) } \\
\hline $0-10 \mathrm{db}$ & $46(39.3)$ & $18(15.4)$ & $53(45.3)$ & $117(100)$ \\
\hline $11-20 \mathrm{db}$ & $29(65.1)$ & $1(2.4)$ & $12(28.6)$ & $42(100)$ \\
\hline $21-30 \mathrm{db}$ & $366(89.7)$ & $33(8.1)$ & $9(2.2)$ & $408(100)$ \\
\hline$>30 \mathrm{db}$ & $83(100)$ & $0(0.0)$ & $0(0.0)$ & $83(100)$ \\
\hline Total & $524(80.6)$ & $52(8.0)$ & 74 (11.4) & $650(100)$ \\
\hline \multicolumn{5}{|c|}{ OU (count, row \%) } \\
\hline $1-10 \mathrm{db}$ & $35(50)$ & $4(5.8)$ & $31(45.0)$ & $70(100)$ \\
\hline $11-20 \mathrm{db}$ & $31(77.5)$ & $1(2.5)$ & $8(20.0)$ & $40(100)$ \\
\hline $21-30 \mathrm{db}$ & $377(94.7)$ & $19(4.8)$ & $2(0.5)$ & $398(100)$ \\
\hline$>30 \mathrm{db}$ & $161(98.2)$ & $3(1.8)$ & $0(0.0)$ & $164(100)$ \\
\hline Total & $602(89.8)$ & $27(4.0)$ & $41(6.1)$ & $672(100)$ \\
\hline
\end{tabular}

$\mathrm{dB}$, decibels; HVF, Humphrey visual fields; OD, right eye; OS, left eye, OU, both eyes.

OS and $0.83 \mathrm{OU}$, indicating that the iPad response of a normal clear image and an abnormal image are separated. The ROC curve with a cut-off value of $23 \mathrm{~dB}$ had a sensitivity of $74 \%$ and specificity of $72 \%$ for OD, a sensitivity of $73 \%$ and specificity of $79 \%$ for OS and a sensitivity of $73 \%$ and specificity of $84 \%$ for OU.

\section{DISCUSSION}

We have successfully designed and tested a novel iPad app that allows glaucoma subjects to self-pictorialise their visual field defects. This innovation fills a gap in the translation of clinicderived automated perimetry used to monitor and treat glaucoma and its real-world impact on patients. For the first time, patients, caregivers and physicians can visualise the glaucomatous field defects from a patient's perspective and better understand the real-world impact of automated perimetry results.

Glaucoma is a 'silent' blinding disease and half of all newly diagnosed glaucoma patients already have moderate to severe vision loss at diagnosis. ${ }^{3} 18$ Glaucoma patients are frequently unaware of their field defects until an advanced stage, perhaps due to the phenomenon of 'filling in'. Filling in, which is the completion of missing information by visual cortex without direct sensory input, is the perceptual mechanism used to describe this inattentiveness to scotomas. ${ }^{9}{ }^{10}$ Filing in has been studied by vision scientists in physiologic scotomas (blind spot), artificial scotomas and animal experiments with destruction of retinal photoreceptors and receptive field mapping in the visual cortex. ${ }^{19}$ The results of these studies are fairly similar-cortical mechanisms appears to 'fill in' the scotoma with surrounding colours, lines, patterns and motion. ${ }^{20}{ }^{21}$ Anatomically, receptive fields of destroyed retinal areas have been shown to spread outside the zone of the lesion. ${ }^{22}$ The most important factors determining the filling in process appear to be (a) eccentricity of scotomas: peripheral scotomas fade easier than central which is explained by the increase in receptive field size with retinal eccentricity, ${ }^{21}$ (b) size of scotomas: if the object is smaller than the scotoma, it is impossible to fill in; instead, the object disappears and if the scotoma is very large, the brain will perceive the area as distorted. ${ }^{9}$ Abadi et al showed human blind spot awareness and filling in occurred only when the stimulus width was more than that of the blind spot, stimulating the functional retina. ${ }^{9}$

Visual perception in subjects with eye diseases has not been studied extensively. Some studies in central macular lesions suggest that filling in does occur across pathologic retinal scotomas with binocularity playing an important role. ${ }^{23}{ }^{24}$ In eyes with bilateral macular lesions, filling in occurred in $85 \%$ of the less severely affected eye. Unilateral macular lesions and the more severely affected eye in binocular retinal lesions do not demonstrate filling in. ${ }^{23}$

Our iPad app allowed our subjects to map out their visual perception in a novel experimental design. All subjects reported that the image they made on the app reflected their visual perception accurately. Subjects were allowed to choose between degrees of 'blur' and 'dimness'. None of the patients mapped out their vision as diminishing contrast or blackness. All mapped out their vision as different degrees of blur. Our experimental study results corroborate patient descriptors of their vision in previous studies. ${ }^{4} 1213$ Our innovation lies in the point-to-point correlation and quantification between HVF thresholds and subjective perceptions at those loci. We tested our app through monocular and binocular testing in all 12 subjects and analysed the 36 responses as three different subsets: right eyes (OD), left eyes (OS) and both eyes (OU). The congruity of results in all three datasets demonstrates the scientific rigour of our study methodology and analysis.

We were able to correlate threshold sensitivity values on the HVF to the subjective response at the corresponding visual angle on the poster. We showed that lower HVF thresholds were associated with increased blur. An independent ROC analysis of all three subsets (OD, OS, OU) found that the threshold cut-off for normal/abnormal visual perception was $23 \mathrm{db}$. Descriptive studies have shown us that glaucoma patients perceive their vision as 'normal' or 'blurred/ missing' ${ }^{12} 13$ The psychophysical studies with physiologic and simulated blind spots described above allow us to infer what the glaucoma patient may see in their areas of defects. ${ }^{4}$

Previous studies have used global measures of visual field to correlate with visual symptoms. ${ }^{25}$ In the Early Manifest Glaucoma trial, a mean deviation worse than $-18 \mathrm{~dB}$ and VFI $<50 \%$ was associated with reduction in vision-related quality of life. ${ }^{26} \mathrm{Hu}$ et al ${ }^{11}$ found that patients with a mean defect of worse than $9.4 \mathrm{~dB}$ on Octopus were more likely to report symptoms of visual field loss. Fujitani et al $^{13}$ reported a mean 10-2 HVF mean deviation value of $17.86 \mathrm{~dB}$ being associated with blurry/missing vision and a mean deviation of $21.18 \mathrm{~dB}$ with black patches. However, previous studies have not correlated subjective visual perception topographically with visual field loss as mapped in clinic like our study does.

Our study has limitations. We included subjects only with good central vision and preserved cognition (high MoCA scores and reliable visual fields). Subjects with advanced cognitive impairment and loss of central vision may have trouble with usage of our app. Each of our subjects performed the test once for each eye, so we did not evaluate test-retest variability and the learning effect. We did not use an eye tracker; subject motivation to maintain fixation is a very important criterion for success of this experiment. Our research focus was pictorialising the glaucoma patient's vision primarily in terms of contrast and blur changes. We did not focus on changes in colour or on vision-related quality of life, awareness of field loss and the emotional impact of disclosing the disability to the glaucoma subjects, which was significant. Despite living with glaucoma for several years, several of 
our subjects and their families were very upset by the degree of their vision loss when it was mapped out on a realistic scene. Future studies will focus on these aspects.

Our iPad app has significant implications for patient and caregiver education through pictorialisation of visual field deficits. There have been artist depictions of visual disability ${ }^{27}$ caused by macular disease (famously by Degas) and cataracts (the later paintings of Monet) but visual disability in glaucoma is not as well appreciated. Hence, the simulations of glaucomatous vision loss as large peripheral black blotches and commas in patient education websites ${ }^{14}$ are inconsistent with this and previous research. Many patients with glaucoma describe the exact moment when they became aware of their visual disability ${ }^{28}$ as an epiphany that allowed them to accept rehabilitative help (personal communication-JS). An iteration of this iPad app that would allow patients to take a picture of their environment and then modify the iPad image of the picture to reflect their perception of the scene is under development. This will have important implications for patient awareness and education of their vision loss. Self-awareness of their visual disability ${ }^{29}$ is essential for motivating glaucoma patients to participate and succeed in a rehabilitation programme.

\section{CONCLUSION}

Glaucoma patients perceive their visual defects as blur and not dimness/black patches as classically described. Simulation of the visual field loss in a naturalistic scene correlated well with clinicbased testing of visual field defects.

Acknowledgements Thanks to Madeline Sharp, Abdullahi Mahamed, Parshav Chauhan and Lyle Reinholz for all their help on the iPad app and Kara Kamerer for graphic design expertise and poster design.

Contributors DG, SK, DA, VG, JS, MN conceived of the study. DG, DA, ZF, DK initiated the study design and JK, MG helped with implementation. The app was developed with the help of DK and ZF. LS and RH provided statistical expertise and conducted the primary statistical analysis. MG, DG wrote the manuscript. All authors contributed to refinement of the study protocol and approved the final manuscript.

Funding National Institute of General Medical Sciences, U54 GM115458, IDeA CTR, Scholars grant, Department of Ophthalmology Pilot Grant, University of Nebraska Medical Center, NIH K23 EY02326.

Competing interests None declared.

Provenance and peer review Not commissioned; externally peer reviewed.

Data availability statement Data are available upon reasonable request.

Supplemental material This content has been supplied by the author(s). It has not been vetted by BMJ Publishing Group Limited (BMJ) and may not have been peer-reviewed. Any opinions or recommendations discussed are solely those of the author(s) and are not endorsed by BMJ. BMJ disclaims all liability and responsibility arising from any reliance placed on the content. Where the content includes any translated material, BMJ does not warrant the accuracy and reliability of the translations (including but not limited to local regulations, clinical guidelines, terminology, drug names and drug dosages), and is not responsible for any error and/or omissions arising from translation and adaptation or otherwise.

Open access This is an open access article distributed in accordance with the Creative Commons Attribution Non Commercial (CC BY-NC 4.0) license, which permits others to distribute, remix, adapt, build upon this work non-commercially, and license their derivative works on different terms, provided the original work is properly cited, appropriate credit is given, any changes made indicated, and the use is noncommercial. See: http://creativecommons.org/licenses/by-nc/4.0/.

ORCID iD

Meghal Gagrani http://orcid.org/0000-0001-7742-1856

\section{REFERENCES}

1 Pascolini D, Mariotti SP. Global estimates of visual impairment: 2010. Br J Ophthalmol 2012:96:614-8.

2 Gupta P, Zhao D, Guallar E, et al. Prevalence of glaucoma in the United States: the 2005-2008 National Health and Nutrition Examination Survey. Invest Ophthalmol Vis Sci 2016;57:2577-85.

3 Gillespie BW, Musch DC, Guire KE, et al. The collaborative initial glaucoma treatment study: baseline visual field and test-retest variability. Invest Ophthalmol Vis Sci 2003;44:2613-20.

4 Hoste AM. New insights into the subjective perception of visual field defects. Bull Soc Belge Ophtalmol. 2003;65-71. PMID: 12784579. Available https://pubmed.ncbi.nlm. nih.gov/12784579/

5 Tanabe S, Yuki K, Ozeki N, et al. The association between primary open-angle glaucoma and motor vehicle collisions. Invest Ophthalmol Vis Sci 2011;52:4177-81.

6 McGwin G, Xie A, Mays A, et al. Visual field defects and the risk of motor vehicle collisions among patients with glaucoma. Invest Ophthalmol Vis Sci 2005;46:4437-41.

7 Baig S, Diniz-Filho A, Wu Z, et al. Association of fast visual field loss with risk of falling in patients with glaucoma. JAMA Ophthalmol 2016;134:880-6.

8 Kaleem MA, West SK, Im LT, et al. Referral to low vision services for glaucoma patients: referral patterns and characteristics of those who refer. J Glaucoma 2017;26:e115-20.

9 Abadi RV, Jeffery G, Murphy JS. Awareness and filling-in of the human blind spot: linking psychophysics with retinal topography. Invest Ophthalmol Vis Sci 2011:52:541-8.

10 Awater H, Kerlin JR, Evans KK, et al. Cortical representation of space around the blind spot. J Neurophysiol 2005;94:3314-24.

$11 \mathrm{Hu}$ CX, Zangalli C, Hsieh M, et al. What do patients with glaucoma see? Visual symptoms reported by patients with glaucoma. Am J Med Sci 2014;348:403-9.

12 Crabb DP, Smith ND, Glen FC, et al. How does glaucoma look?: patient perception of visual field loss. Ophthalmology 2013;120:1120-6.

13 Fujitani K, Su D, Ghassibi MP, et al. Assessment of patient perception of glaucomatous visual field loss and its association with disease severity using Amsler grid. PLoS One 2017;12:e0184230.

14 Glaucoma Vision Simulator. American Academy of Ophthalmology. 2019. Available https://www.aao.org/eye-health/diseases/glaucoma-vision-simulator (accessed 14 Jan 2020)

15 Allingham RR, Freedman S, Damji K, et al., eds. Shield's textbook of glaucoma. 5th edn. Lippincott Williams \& Wilkins, 2004.

16 Nelson-Quigg JM, Cello K, Johnson CA. Predicting binocular visual field sensitivity from monocular visual field results. Invest Ophthalmol Vis Sci 2000;41:2212-21. PMID: 10892865. Available https://pubmed.ncbi.nlm.nih.gov/10892865/

17 Bengtsson B, Heijl A. A visual field index for calculation of glaucoma rate of progression. Am J Ophthalmol 2008;145:343-53.

18 Boodhna T, Crabb DP. Disease severity in newly diagnosed glaucoma patients with visual field loss: trends from more than a decade of data. Ophthalmic Physiol Opt 2015;35:225-30.

19 Darian-Smith C, Gilbert CD. Topographic reorganization in the striate cortex of the adult cat and monkey is cortically mediated. J Neurosci 1995;15:1631-47.

20 Ramachandran VS. Filling in gaps in perception: part I. Curr Dir Psychol Sci 1992;1:199-205.

21 Ramachandran VS. Filling in gaps in perception: part II. Scotomas and phantom limbs. Curr Dir Psychol Sci 1993;2:56-65.

22 Gilbert CD. Plasticity in visual perception and physiology. Curr Opin Neurobiol 1996:6:269-74.

23 Cohen SY, Lamarque F, Saucet J-C, et al. Filling-in phenomenon in patients with age-related macular degeneration: differences regarding uni- or bilaterality of central scotoma. Graefes Arch Clin Exp Ophthalmol 2003;241:785-91.

24 Crossland MD, Bex PJ. Spatial alignment over retinal scotomas. Invest Ophthalmol Vis Sci 2009;50:1464-9.

25 McKean-Cowdin R, Varma R, Wu J, et al. Severity of visual field loss and health-related quality of life. Am J Ophthalmol 2007;143:1013-23.

26 Peters D, Heij A, Brenner L, et al. Visual impairment and vision-related quality of life in the early manifest glaucoma trial after 20 years of follow-up. Acta Ophthalmol 2015:93:745-52.

27 Marmor MF. Ophthalmology and art: simulation of monet's cataracts and Degas' retinal disease. Arch Ophthalmol 2006;124:1764-9.

28 Home. Going blind. Available http://goingblindmovie.com/ (accessed 14 Jan 2020)

29 Port A, Willmott C, Charlton J. Self-awareness following traumatic brain injury and implications for rehabilitation. Brain Inj 2002;16:277-89. 